\title{
8th European Breast Cancer Conference (EBCC-8), Vienna, March 21-24, 2012
}

\author{
Michael Gnant ${ }^{\mathrm{a}, \mathrm{b}} \quad$ Birgit Beermann ${ }^{\mathrm{b}}$ \\ aDepartment of Surgery, \\ ${ }^{\mathrm{b}}$ Comprehensive Cancer Center, Medical University of Vienna, Austria
}

Prof. Dr. med. Michael Gnant, MD, FACS

Medizinische Universität Wien, Universitätsklinik für Chirurgie

Comprehensive Cancer Center Vienna

Währinger Gürtel 18-20,1090 Wien, Austria

Tel. +43 1 40400-5646, Fax -7603

michael.gnant@meduniwien.ac.at

For the 8th European Breast Cancer Conference (EBCC) 4,000 international scientists, physicians, patient advocates, and industry representatives convened at the Austria Centre Vienna. In an intensive exchange of interdisciplinary experts involving a variety of popular formats (state-of-the-art lectures, clinical science symposia, debates, proffered paper sessions, etc.), the newest developments in breast cancer research and treatment were presented and discussed. A record number of abstract submissions were received for the EBCC, and an international peer review committee found an interesting selection covering all important aspects of breast cancer research, including diagnosis and screening, local and systemic treatment, lifestyle issues and structural aspects of multidisciplinary breast health centres.

Most importantly - and demonstrating the successful implementation of the EBCC as a major international conference - original research was presented for the first time at the EBCC. Reflecting the important participation of patient advocates in program creation and conference planning, summary sessions were held in order to reflect the potential of newly presented original research in tomorrow's daily clinical practice. The luckily perfect spring weather in Vienna promoted continuing discussions and exchange of experiences among participants also between and after the well-attended scientific sessions.

As a new but highly successful feature, a local 'patient day' was organized in Vienna on the final day of the EBCC with the aim of translating the latest research findings into understandable language for patients, patients advocates, and the interested public, who attended in numbers.

\section{Important Research Highlights Presented at EBCC-8}

\section{New Application Method of Trastuzumab}

Results from the HannaH study in women with HER2-positive early breast cancer (eBC) showing for the first time that a new way of giving trastuzumab by subcutaneous (s.c.) injection leads to comparable efficacy to the current way of giving the medicine by intravenous (i.v.) route. Trastuzumab s.c. may provide greater convenience to patients versus the traditional i.v. method due to its less invasive administration route and quicker administration time (5 versus 30-90 min). HannaH is a phase III, randomised, open-label, international, multicentre study. A total of 596 patients with operable or locally advanced eBC were enrolled. The study investigated the efficacy, pharmacokinetics and safety of trastuzumab s.c. (in a ready-to-use vial) and trastuzumab i.v. in the neoadjuvant/adjuvant treatment setting of women with HER2-positive eBC. Participants in the trastuzumab s.c. arm received: $600 \mathrm{mg}$ (fixed dose) of trastuzumab s.c. plus chemotherapy for 8 cycles before surgery/ trastuzumab s.c. alone for 10 cycles after surgery. Participants in the trastuzumab i.v. arm received: an initial $8 \mathrm{mg} / \mathrm{kg}$ body weight loading dose of trastuzumab i.v. followed by $6 \mathrm{mg} / \mathrm{kg}$ maintenance dose both in combination with chemotherapy for a total of 8 cycles before surgery, as per the standard i.v. regimen/trastuzumab i.v. alone for 10 cycles after surgery. Treatment duration was 1 year in total for both arms. Efficacy, determined by pathologic complete response, in patients treated in the s.c. arm was in the same range as in patients who received the i.v. formulation (45.4 and $40.7 \%$, respectively). The co-primary endpoints of pharmacokinetic and efficacy met their pre-specified criteria. The drug concentration in the blood measured just before

\section{KARGER}

Fax +497614520714

Information@Karger.de

www.karger.com 
surgery was at least as high for the s.c. as for the i.v. formulation ( 69.0 and $51.8 \mu \mathrm{g} / \mathrm{ml}$, respectively). This is important in order to demonstrate comparable efficacy. Adverse events (AEs) in the study were consistent with the known trastuzumab safety profile. No new safety signals were identified. Overall, the incidence of most common AEs (above 10\% in either arm) was comparable. Severe AEs (grade $>3$ ) occurred at a similar incidence between arms (52\% for trastuzumab i.v. and $51.9 \%$ for trastuzumab s.c.). Cardiac AE were similar in both treatment arms: 12.1 vs. $11.4 \%$ in the i.v. and s.c. arms, respectively. $11 \%$ of patients who received the s.c. injection experienced an injection site reaction (most commonly pain at the site of injection) which was mild in intensity in $95 \%$ of patients.

Best Abstract: Jakisch C, et al. Subcutaneous administration of trastuzumab in patients with HER2-positive early breast cancer: results from the phase III randomised, open-label, multi-centre neoadjuvant-adjuvant HannaH study.

\section{Intraoperative Ultrasound to Optimize Breast Conserving Surgery}

The use of ultrasound-guided surgery (USS) to remove tumours from women who have palpable breast cancer is much more successful than standard surgery in excising all the cancerous tissue while sparing as much healthy tissue as possible, according to the results of a randomised controlled trial. Nicole Krekel, a PhD student from Amsterdam and her colleagues randomised 124 patients with palpable early stage breast cancer to either USS or standard palpation-guided surgery (PGS). They found that among the 61 women in the USS group only $3.3 \%$ of the margins contained cancerous cells, compared with $16.4 \%$ among the 63 women in the PGS group. As a result, USS resulted in a significant reduction in re-excisions, mastectomies, and extra radiotherapy. The amount of healthy tissue removed in the USS group was smaller than in the PGS group (40 vs. $58 \mathrm{~cm}^{3}$ ). By measuring the volume of the tissue excised during surgery and the actual tumour diameters, the researchers calculated the amount of excess healthy tissue that had been removed, which gave them the 'calculated resection ratio' (CRR). A CRR of 1.0 would indicate the ideal amount of tissue had been removed, while a CRR of 2.0 would indicate that double the amount necessary had been removed. They found that the CRR in the USS group was 1.0 (ideal), while in the PGS group it was 1.9. By using USS, $96.7 \%$ of all tumours were excised with adequate, tumour-free margins, compared with only $83.4 \%$ in the PGS group, yet the volume of tissue excised was significantly smaller in the USS group.

Late-breaking abstract 1: Krekel N, et al. Intra-operative ultrasound is imperative to obtain adequate tumour margins and excision volumes in breast-conserving surgery for palpable breast cancer: results of a randomised controlled trial.

\section{Addition of Exemestane to Fulvestrant Makes no Difference} Optimal endocrine treatment for postmenopausal women with advanced oestrogen receptor positive breast cancer progressing on a non-steroidal aromatase inhibitor (AI) is un- clear. The EFECT study showed no difference in efficacy between the steroidal antioestrogen fulvestrant and the steroidal AI exemestane in this setting. Pre-clinical data suggest fulvestrant may be more effective in a low-oestrogen environment. 2 recent trials of fulvestrant combined with anastrozole compared with anastrozole alone in first-line setting gave mixed results. In SoFEA, a multicentre randomised partially blinded phase III study, postmenopausal women were allocated to fulvestrant $250 \mathrm{mg}$ monthly with $500 \mathrm{mg}$ loading dose plus anastrozole $1 \mathrm{mg}$ daily (fulvestrant/anastrozole $n=243)$, fulvestrant plus placebo $(n=231)$ or exemestane $25 \mathrm{mg}$ daily $(\mathrm{n}=249)$. Patients should have responded to previous non-steroidal AI in metastatic setting for more than 6 months or received more than 12 months non-steroidal AI as adjuvant treatment and should be progressing on non-steroidal AI at trial entry. Primary endpoint was progression-free survival (PFS). 723 patients at a median age of 64 years were enrolled from 82 centres in the UK and 4 in South Korea. Prior AI had been given as adjuvant treatment to $18 \%$ of patients for a median of 27.9 months, and to $82 \%$ of patients for locally advanced/metastatic disease for a median of 19.3 months. Median PFS was 4.4 months for the combination fulvestrant/anastrozole, 4.8 months for fulvestrant, and 3.4 months for exemestane. As expected, longer PFS was positively correlated with duration of prior AI exposure but no interaction with treatment was observed. No differences were observed for objective response rate, clinical benefit rate and overall survival. $90.4 \%$ of patients had serum oestradiol levels below $3.0 \mathrm{pmol} / \mathrm{l}$ at baseline, while at 3 months mean values differed as expected between patients treated with fulvestrant/anastrozole $(2.8 \mathrm{pmol} / \mathrm{l})$ and fulvestrant $(15.0 \mathrm{pmol} / \mathrm{l})$, confirming oestrogen suppression with fulvestrant/anastrozole. Treatment was well tolerated: serious adverse events were rare. The authors concluded that SoFEA provides no evidence that fulvestrant combined with anastrozole (with fulvestrant loading dose) is more effective than fulvestrant or exemestane alone in patients with acquired resistance to nonsteroidal AI. Median PFS in SoFEA is similar to EFECT, and the lack of added benefit for the combination therapy is consistent with FACT.

Late-breaking abstract 2: Johnston S, et al. Fulvestrant alone or with concomitant anastrozole vs exemestane following progression on non-steroidal aromatase inhibitor - first results of the SoFEa trial.

\section{Overcoming Hormone Resistance}

The latest results from BOLERO-2, a multinational, doubleblind, placebo-controlled, phase III clinical trial, evaluating a new treatment for breast cancer in post-menopausal women with advanced oestrogen receptor positive breast cancer that is resistant to hormonal therapy show that the combination of 2 cancer drugs, everolimus and exemestane, significantly improves bone strength and reduces the chances of cancer metastasising in the bone. BOLERO-2 had shown previously that the combination of the 2 drugs significantly improved outcome, stopping further tumour growth for nearly 11 
months, in a group of patients with a form of breast cancer that is highly resistant to treatment. However, as some anticancer drugs are associated with reduced bone mineral density and an increased risk of fractures, it was important to discover whether everolimus and exemestane, used with or after treatment with other drugs such as non-steroidal AI (e.g. anastrozole), affected bone strength. The BOLERO-2 trial provided an opportunity to look at markers for bone turnover and bone resorption in the 724 patients enrolled in the trial and randomised to receive either everolimus and exemestane or exemestane and placebo. Patients had an average age of 62 years, were from 24 different countries and had been treated previously with AI. The researchers found that levels of all 3 bone markers decreased significantly after 6 and 12 weeks for women taking everolimus, indicating a low turnover of bone, which improves bone strength and health. After 6 weeks, bone-specific alkaline phosphatase (BSAP) had dropped by $5.5 \%$, amino-terminal propeptide of type 1 collagen (P1NP) had dropped by $20.4 \%$, and C-terminal crosslinking telopeptide of type I collagen (CTX) had dropped by $6.3 \%$. After 12 weeks, they had decreased by 3.6, 26.8 and $0.5 \%$, respectively. In the placebo group they all increased. Overall, out of all the women in the trial, only $3 \%$ of the women taking everolimus had further bone metastases after 60 days, compared with $6 \%$ in the placebo group; in a subgroup of women who were known to have bone metastases at the start of the trial, everolimus halved the rate of further bone metastases, with bone metastases progressing in nearly $4 \%$ of these women, compared to $8 \%$ in the placebo group. This trend continued for longer than 6 months. Any bone-related side effects were rare and those that did occur were of a low grade, including bone pain and fractures.

Late-breaking abstract 3: Gnant M, et al. Everolimus added to exemestane reduced bone markers and disease progression in bone in postmenopausal women with advanced breast cancer: updated results from the BOLERO-2 trial.

\section{Other Important Research Findings and Messages from EBCC-8}

\section{Screening Decreased Breast Cancer Deaths}

The Dutch population-based mammography breast cancer screening programme began in 1989. Jacques Fracheboud, a senior researcher at the Erasmus University Medical Center (Rotterdam, The Netherlands), reported on its first 20 years. Compared with the pre-screening period 1986-1988, deaths from breast cancer among women aged 55-79 fell by 31\% in 2009. Fracheboud: 'We found there was a significant change in the annual increase in breast cancer deaths: before the screening programme began, deaths were increasing by $0.3 \%$ a year, but afterwards there was an annual decrease of $1.7 \%$. This change also coincided with a significant decrease in the rates of breast cancers that were at an advanced stage when first detected.' Between 1990 and 2009, 16.6 million personal invitations were sent to 3.6 million women aged between 50 and 75 years. Overall attendance was $80 \%$, increasing from $73.5 \%$ in 1990 to $81.5 \%$ in 2009 . In the same period, 13.2 million screening examinations were performed among 2.9 million women, resulting in 178,490 (1.35\%) referral recommendations, 95,757 $(0.72 \%)$ biopsies and 66,562 (0.5\%) breast cancer diagnoses. Referral rates increased by approximately 200\% in initial screenings between 1990-1997 and 2005-2009, and by approximately $100 \%$ in subsequent screenings, resulting in an increase in detection rates of more than $30 \%$. For a woman who was 50 in 1990 and who had 10 screenings over the 20 -year period, the cumulative risk of a false positive result was $6 \%$. Over-diagnosis was limited to $2.8 \%$ of all breast cancers diagnosed in the total female population and $8.9 \%$ of screen-detected breast cancers. The total annual cost of the programme was $€ 51.7$ million in 2009 , or $€ 56.65$ per examination. Adjusted for inflation, a breast screening cost approximately $€ 3.50$ less than in 1996. In addition, the screening programme was accurate, with high sensitivity and specificity. Up to 2005 , sensitivity was $74.3 \%$ for initial and $67.6 \%$ for subsequent screens, and the sensitivity was 99 and $99.4 \%$, respectively. (Abstr. 26)

\section{Screening is More Effective than Adjuvant Therapy in Reducing Overall Breast Cancer Mortality}

A Dutch study added further fuel to the debate about whether or not breast cancer screening does more harm than good. The Dutch researchers found that adjuvant therapy reduced deaths by an estimated $13.9 \%$ in 2008 compared to no treatment; but they also found that screening every 2 years reduced deaths by an additional 15.7\%. Rianne de Gelder, a PhD student and researcher at the Erasmus University Medical Center (Rotterdam, The Netherlands), told the conference that she and her colleagues had used a computer modelling technique called microsimulation to show that adjuvant treatment reduced deaths from breast cancer from 67.4 per 100,000 women years to 57.9. With the addition of 2-yearly screening between the ages of 50 and 75 , the deaths fell to 48.8 per 100,000 women years, meaning that adjuvant therapy combined with screening reduced deaths by a total of $27.4 \%$. If screening were to be extended to women aged between 40 and 49 , deaths would be reduced by a further $5.1 \%$, making a total reduction in breast cancer deaths of $31.1 \%$ compared to a situation where there was no treatment and no screening for women aged between 40 and 75 . The researchers assessed the reduction in breast cancer deaths for the total female population over the whole of life (0-100 years), including women who had never been screened. De Gelder said: 'Our study shows that, even in the presence of adjuvant therapy, mammography screening between age 50 and 75 is highly effective in reducing breast cancer deaths - and, in fact, is slightly more effective than adjuvant treatment. It is important to note that our study demonstrated that the observed reduction in breast cancer mortality in The Netherlands could not fully be explained by mammography screening and adjuvant therapy 
only. Other, unknown causes are likely to have contributed to fewer deaths as well.' (Abstr. 28)

\section{MRI Is Most Cost-Effective in Sub-Group of Patients}

Adding magnetic resonance imaging (MRI) to standard breast cancer screening approaches is expensive, though it could be cost-effective for a group of women who may not have inherited the breast cancer susceptibility genes, but who have a familial risk of developing the disease. This is the conclusion of a group of researchers, who conducted a cost-effectiveness analysis of 1,597 women enrolled in the Dutch MRI Screening Study between 1999 and 2007, who had an estimated cumulative lifetime risk of between $15-50 \%$ for developing breast cancer before the age of 70 . The women were screened by way of a clinical breast examination every 6 months and an annual mammography and MRI between the ages of 25 and 70 . 'We found that it costs approximately 3 times as much to add MRI to the screening process for every estimated 1 year of life saved,' said Sepideh Saadatmand, who is a physician and $\mathrm{PhD}$ student at the Erasmus University Medical Center (Rotterdam, The Netherlands). 'When women were screened using clinical breast examinations, mammography and MRI, the cost per detected cancer was about $€ 103,000$. We predicted that screening women between the ages of 35 and 60 in this way would reduce deaths from breast cancer by $24 \%$, at a cost per year of life gained of $€ 30,000$. However, if these women were screened by annual mammography and clinical breast examination alone, the estimated reduction in deaths was $20 \%$, at a cost per year of life gained of $€ 10,000$. 'It is clear that adding MRI to screening programmes for all women with a cumulative life time risk of $15-50 \%$ for breast cancer is highly effective, but possibly too expensive. However, it may be cost-effective for a select subgroup. The subgroups we expect to benefit from MRI screening are women with a cumulative lifetime risk above $20 \%$ due to their family history, who have very dense breast tissue. There is a multi-centre randomised controlled trial running in The Netherlands to investigate this.' (Abstr. 27)

\section{Dense Breasts Nearly Double Relapses}

Until now, little was known about the association between density, tumour characteristics and prognosis once cancer had occurred, and results were conflicting. A Swedish study presented Wednesday in a poster session is one of the largest studies to date studying mammographic density, tumour characteristics and prognosis, including almost $50 \%$ of all Swedish breast cancer cases diagnosed 1993-1995. Louise Eriksson and her colleagues from the Karolinska Institutet (Stockholm, Sweden) found that women with denser breasts had nearly double the risk of their cancer recurring, either in the same breast or in the surrounding lymph nodes, than women with less dense breasts. They warn that doctors should take breast density into account when making decisions about treatment and follow-up for these women. The researchers studied the mammograms and outcomes for 1,774 postmenopausal women who were aged 50-74 and who were part of a larger study of all women with breast cancer diagnosed between 1993 and 1995 in Sweden. They found that if a woman has a percentage density (PD) at diagnosis of $25 \%$ or more, she has an almost 2-fold increased risk of local recurrence in the breast and surrounding lymph nodes than women with a PD of less than $25 \%$. However, density does not increase the risk of distant metastasis and has no effect on survival. And although mammographic density was one of the strongest risk factors for breast cancer it does not seem to influence tumour development in any specific way but seems to act as a general stimulator of tumour development. (Abstr. 131)

\section{Pregnancy Is Not Harmful for Survivors \\ of Endocrine-Responsive Breast Cancer}

New research has shown for the first time that it is safe for women who have been diagnosed with oestrogen receptor positive breast cancer to become pregnant. The findings suggest that becoming pregnant at any time following a diagnosis of breast cancer does not increase the risk of recurrence, even if the pregnancy occurs during the first 2 years after diagnosis. Furthermore, patients who become pregnant appear to survive longer than those who do not. Hatem A. Azim, a medical oncologist at the Jules Bordet Institute (Brussels, Belgium), and colleagues carried out a study in several countries in which they enrolled 333 women who had become pregnant at any time following a breast cancer diagnosis and matched them with 874 similar breast cancer patients who did not become pregnant. Importantly, the study included only women whose oestrogen receptor status (positive or negative) and disease outcome were known. In addition, women in the control group had not relapsed at the time the matched case became pregnant; this enabled the researchers to adjust as far as possible for the 'healthy mother effect' - a phenomenon where the results could be influenced by the fact the women who became pregnant might be healthier than breast cancer patients in the control group. The researchers followed the women for an average of 4.7 years after pregnancy. During this period, $30 \%$ of the total of 1,207 women had a recurrence of their disease. (Abstr. 21)

\section{Genomic Test Spares Chemotherapy}

Sabine Linn, Medical Oncologist at The Netherlands Cancer Institute, Amsterdam, presented the first study where a genomic test has been incorporated in decision-making about adjuvant systemic therapy. The study aimed to assess the feasibility of implementing Mammaprint ${ }^{\circledR}$, a test for a selection of 70 genes in a tumour, in daily clinical practice in The Netherlands, as well as its effect on adjuvant systemic treatment decisions. The researchers studied follow-up data from 427 patients with early breast cancer who had taken part in a study called RASTER (MicroarRAy prognoSTics in breast cancER). Their cancers had not yet spread to the lymph 
nodes. In addition to the results of the Mammaprint test, the researchers retrospectively used the risk prediction tool Adjuvant!Online (AOL) for comparison. In around 35\% of the patients the results of the 2 tests were discordant. Treatment decision-making was based on the result of the Mammaprint test, Dutch national guidelines and patients' and doctors' preferences. In the group classified as low-risk by the Mammaprint test only $15 \%$ of the 219 patients received adjuvant chemotherapy as opposed to $81 \%(169 / 208)$ in the group classified as high-risk by the Mammaprint test. The first group had a 5-year distant disease-free survival (DDFS) rate of 96\% compared with $90 \%$ in the high-risk group. In the groups where the Mammaprint and AOL results were discordant, where the genomic test predicted a low risk and AOL a high risk, $43 \%$ of patients received endocrine therapy and $24 \%$ chemotherapy. There was a $98 \%$ DDFS in this group. Where AOL predicted a low risk but Mammaprint a high one, 78\% received endocrine therapy and $57 \%$ chemotherapy. The DDFS in this group was $95 \%$. Based on these data, the use of the genomic test could lead to a reduction of nearly $30 \%$ in the use of adjuvant chemotherapy without compromising patient outcomes. (Abstr. 207)

\section{Long-Time Protective Effect of Radiotherapy for DCIS}

Radiotherapy treatment (RT) after surgery for ductal carcinoma in situ (DCIS) still has a major protective effect against recurrence more than 15 years later, according to the results of an international trial. Mila Donker, a research physician from the Netherlands Cancer Institute, Antoni van Leeuwenhoek Hospital (Amsterdam, The Netherlands), worked with colleagues from 13 other countries under the auspices of the European Organisation for the Research and Treatment of Cancer (EORTC), based in Brussels, Belgium. They analysed the 15-year follow-up of more than 1,000 DCIS patients, 50\% of whom had received RT after total surgical excision of a tumour of less than $5 \mathrm{~cm}$ diameter and $50 \%$ who had not. When radiotherapy had not been given, almost 1 in 3 women had developed a local recurrence, while the $50 \%$ risk reduction in those patients who had had RT held true for both an in-situ and an invasive cancer recurrence, where the cancer had spread outside the duct. The 15-year cumulative incidence for DCIS recurrence in the surgery group was $14.9 \%$, as opposed to $7.5 \%$ in those patients in the combined group (surgery plus RT), and for an invasive recurrence the rates were $15.5 \%$ (surgery only) and $9.8 \%$ (combined). Although no survival difference was seen between the surgery alone and the surgery plus RT group, women who had an invasive recurrence had a significantly worse survival compared with those who had a DCIS recurrence or no recurrence at all. (Abstr. 217)

Genetic Variation Correlates with Chemotherapy Side Effects The largest study ever on the effect of genetic variability on the toxicity of chemotherapy in breast cancer shows that it is possible to predict which patients are most likely to suffer serious side effects. Christof Vulsteke, from the Catholic University of Leuven, Belgium, and his team examined germline DNA from blood samples from 1,089 breast cancer patients who were treated between 2000 and 2010 with 3 commonly used chemotherapy drugs (fluorouracil, epirubicin and cyclophosphamide). For each patient, the variability in the genes that are important for metabolising these 3 chemotherapeutic drugs was compared with the side effects experienced. 'We found that genetic variation in one gene was highly correlated with chemotherapy side effects,' said Vulsteke. 'Investigating this gene before starting chemotherapy would allow us to support the patient with either growth factors to increase the patient's immunity, or dose modifications, or a different chemotherapy regimen better adapted to the patient, or a combination of these.' The researchers now intend to look for data from other European countries in order to further validate their results. They will also continue to follow up on their own patients; currently the patients are followed up, on average, for 5 years. (Abstr. 237)

\section{Circulating Tumour Cells Can Be Used for Prediction of Prognosis}

Results from the first large-scale study of the relevance of circulating tumour cells (CTCs) in early breast cancer prognosis show that patients with at least 5 CTCs detected straight after surgery have a 4-fold increase in risk of recurrence and a 3 -fold increase in risk of death. Bernadette Jäger, from the Department of Gynecology and Obstetrics - Campus Innenstadt, Ludwig Maximilian University Hospital (Munich, Germany), and colleagues from 9 German university hospitals analysed the numbers of CTCs in the blood of 2,026 patients in a trial called SUCCESS A. The patients had all had a complete resection of their primary tumour prior to commencing chemotherapy. CTCs were detected in $21.5 \%$ of them, a much lower rate than that which is usually seen in metastatic breast cancer. Detecting the presence of circulating tumour cells (CTCs) in the blood of women with early breast cancer after surgery but before the start of chemotherapy can provide useful information about their chances of surviving the disease. CTCs are cancer cells which are detectable in patients with a solid tumour and their value in the prognosis of metastatic breast cancer has been known for a few years. Until now, however, there has been little information about their role in early disease. (Abstr. 301)

\section{Obesity Is a Risk Factor of Breast Cancer Recurrence \\ Also with Respect to Chemotherapy}

Women who are overweight or obese when they are diagnosed with breast cancer are at higher risk of cancer recurrence or related death than are leaner women, according to a new study presented at the EBCC-8. This finding held true even though the study mandated that chemotherapy dosage be adjusted for body weight, and adds further to the evidence 
that lifestyle factors can influence cancer prognosis. Jennifer Ligibel, a medical oncologist at the Dana-Farber Cancer Institute (Boston, USA), and colleagues, studied data from 1,909 patients who were enrolled into a study called CALGB 9741 between 1997 and 1999. The study was set up to investigate different dosing schedules for adjuvant chemotherapy in patients with node-positive cancer. After extracting height and weight data from the patient records, they went on to evaluate the relationship between body mass index (BMI) with relapse-free survival (RFS) and overall survival (OS). 1.2\% of the patients were underweight, $32.6 \%$ normal weight, $32.9 \%$ overweight and $33.3 \%$ obese. $49 \%$ of patients were menopausal, $65 \%$ had oestrogen-receptor positive cancers, and $70 \%$ received the oestrogen-receptor blocking treatment tamoxifen. BMI was related to both RFS and OS; for example, the 10 -year RFS of a patient who was overweight was $70 \%$; compared with $65 \%$ for one who was obese. (Abstr. 413)

\section{Clinical Benefit for Fulvestrant plus Goserelin in Premenopausal Women}

A pilot study has evaluated the combination of the pure antioestrogen fulvestrant with the LHRH-agonist goserelin in a small subgroup of patients: in premenopausal women with hormone receptor (HR)-positive metastatic breast cancer. More than every second patient showed a meaningful clinical benefit. Rupert Bartsch and colleagues from the Comprehensive Cancer Center Vienna (Austria) have now investigated the feasibility and safety of combining fulvestrant, a pure antioestrogen, with goserelin, an LHRH-agonist, in premenopausal women with HR-positive metastatic breast cancer. Pretreated premenopausal patients with metastatic breast cancer eligible for on-going endocrine treatment received fulvestrant $250 \mathrm{mg}$ and goserelin $3.6 \mathrm{mg}$ every 4 weeks as first- to fourthline therapy. Clinical benefit rate (CBR) was defined as primary study endpoint. Based upon previous data, a CBR of $50 \%$ was considered to indicate meaningful clinical activity. 26 patients received treatment as scheduled. $81 \%$ were pretreated with tamoxifen and $69 \%$ had received prior aromatase inhibitors in combination with goserelin. The majority of patients $(69 \%)$ presented with visceral metastases. Complete response was observed in a single patient, partial response in 3 patients, and disease stabilisation of 6 months or more in 11 patients. This translated into a clinical benefit rate of $58 \%$. Treatment with fulvestrant and goserelin was well tolerated, and none of the patients discontinued therapy due to toxicity. No instances of grade 4 toxicity were recorded; grade 3 toxicities consisted of 2 cases. Bartsch and colleagues concluded that the combination therapy offers meaningful activity in premenopausal patients with HR-positive metastatic breast cancer. (Abstr. 45)

\section{Non-Drug Therapy against Drug-Induced Menopause}

Researchers found that the menopausal symptoms caused by giving chemotherapy or hormonal therapy to younger women with breast cancer can be ameliorated considerably through the use of cognitive behavioural therapy (CBT) and physical exercise (PE). 'This is the first study to investigate the efficacy of these 2 interventions specifically in women who have experienced acute, treatment-induced menopause,' said Marc van Beurden from The Netherlands Cancer Institute, Amsterdam. The researchers studied 422 breast cancer patients with an average age of 48 years recruited from 14 hospitals of the Netherlands. They were randomly assigned to 4 groups - CBT alone, PE alone, CBT and PE combined - and a control group. Compared with the control group, all those patients who received one or both interventions showed an overall decrease in the levels of menopausal symptoms, in addition to reporting an increase in sexuality and an improvement in physical functioning. These positive effects were still apparent after 6 months. CBT is an action-oriented form of psychosocial therapy and psycho-education that focuses on changing an individual's thoughts (cognitive patterns) in order to change his or her behaviour and emotional state. In the current study, CBT was combined with relaxation techniques. The CBT programme consisted of 6 weekly group sessions of 90 min each, including relaxation exercises. The primary focus of CBT was on hot flushes and night sweats, but other symptoms were also addressed. The PE programme was a 12-week, individually tailored, home-based and self-directed exercise programme of 2.5-3 h per week. Initial training and follow-up was provided by physiotherapists. The goal was to exercise at an intensity level that achieved a target heart rate. While the evidence that the interventions worked was convincing, compliance with them was poor, the researchers say. In the case of CBT, it was difficult to schedule the group sessions at a time that was convenient for women who often had both work and parenting responsibilities. The frequency and intensity of the PE programme was also a challenge for many women. (Abstr. 199)

\section{Update on Trials 'in Progress'}

\section{Neoadjuvant Therapy with EC or AC or FEC Followed by Nanoparticle Albumin-Bound Therapy}

A phase II trial evaluates neoadjuvant anthracycline based regimens followed by a combination of nanoparticle albuminbound paclitaxel and trastuzumab. The study is aimed at patients with operable T1c-3, N0-1, HER2-positive breast cancer. According to investigator Mitsuhiko Iwamoto from Osaka Medical College (Japan), patients are treated with 4 cycles of one of the following 3 regimens: neoadjuvant EC or AC or FEC every 21 days, followed by a combination of nanoparticle albumin-bound paclitaxel $\left(260 \mathrm{mg} / \mathrm{m}^{2}\right)$ and trastuzumab. Patients undergo surgery 4-6 weeks after completion of chemotherapy. Primary endpoint is $\mathrm{pCR}$, defined as no evidence of invasive tumours in the final surgical sample both in the breast and axillary lymph nodes. 5 patients have 
been included since the start of the study. A total of 45 patients will be recruited by the last quarter of 2013 .

\section{A New Biomarker, 'Brother of the Regulator of Imprinted Sites' (BORIS)}

This potentially clinically useful biomarker has been identified in leukocytes of breast cancer patients and is currently being evaluated as a screening tool to diagnose cancer early in women with familial breast cancer. Anuradha Apte, Essex County Hospital, UK, and colleagues have discovered that the protein BORIS, appears in leukocytes of breast cancer patients. In 2011, 129 women were recruited. Western blot analysis showed low levels of BORIS expression in 10 women. Immunocytochemistry showed that 9 out of these 10 participants had an immuno-reactivity score (IRS) ranging between 0 and 1.6. 1 participant had an IRS score of 2.4. This participant had a positive mammogram and was histologically diagnosed with invasive ductal carcinoma of the breast. Clinically, mammograms of all other participants were negative. The aim of the ongoing study is to recruit 300 women (aged 40-50 years) with high risk of familial breast cancer. Blood samples $(10 \mathrm{ml})$ are collected from each participant every year for 5 consecutive years at the time of their annual mammogram. BORIS expression in the leukocytes is evaluated by various methods including immunocytochemistry, Western blot and ELISA. The results are compared with the corresponding mammograms. If a woman shows an obvious increase in her BORIS level and her corresponding mammogram is negative, she is offered an MRI scan to confirm the presence or absence of the disease.

\section{The RESILIENCE Trial: Sorafenib plus Capecitabine} as First- or Second-Line Therapy in HER2-Negative Advanced Breast Cancer Patients

This is an ongoing multinational, double-blind, placebo-controlled, phase III trial designed to assess sorafenib plus capecitabine as first- or second-line therapy in advanced HER2-negative BC. Eligibility criteria include: age $\geq 18$ years; 1 or less prior chemotherapy regimens for advanced $\mathrm{BC}$; resistant to/failed taxane and anthracycline or no indication for further anthracycline; no prior VEGF treatment. Patients are randomised to capecitabine $\left(1,000 \mathrm{mg} / \mathrm{m}^{2}\right.$ PO BID, days 1-14 of 21) with sorafenib (PO BID, days 1-21, total dose $600 \mathrm{mg} /$ day) or placebo. Capecitabine and sorafenib/placebo doses can be escalated to $1,250 \mathrm{mg} / \mathrm{m}^{2}$ BID and $800 \mathrm{mg} /$ day, respectively, as tolerated. The protocol outlines strategies to manage toxicities with dose interruption and reduction. Dose re-escalation after reduction is only allowed for sorafenib/ placebo. Radiographic assessment is scheduled every 6 weeks for 36 weeks, afterwards every 9 weeks. The primary endpoint of RESLIENCE is PFS. Secondary endpoints include overall survival, time to progression, overall response rate, and duration of response. Enrolment began in November 2010. Targeted enrolment is 519 patients.

\section{Conclusion}

Given the huge amount of positive feedback the organizers have received from EBCC- 8 participants, and the large number of participants and abstracts submitted, the Early Breast Cancer Conference continues to be a successful enterprise, particularly in a changing funding environment that makes huge international conferences more difficult to organize than ever. EORTC, EUSOMA, and Europa Donna as pillars of the EBCC-8 organization, together with the experienced ECCO conference team were rewarded for their efforts and work by the acceptance of EBCC as the most important breast cancer conference in Europe. The next conference (EBCC-9) will be held in Glasgow, Scotland, from March 21 to $23,2014$.

\section{Acknowledgements}

We appreciate the great work by the EBCC-8 organizing team in making EBCC-8 a huge success. In particular, the ECCO media team around Mary Rice and Emma Mason contributed to this by excellent text and media support. 